\title{
Commentary on "The Perception and Cognition of Time in Balinese Music” by Andrew Clay McGraw
}

\author{
IAN CROSS, SATINDER GILL, SARAH KNIGHT, CHRIS NASH, \\ TAL-CHEN RABINOWITCH, LYDIA SLOBODIAN, NETA SPIRO, \\ GHOFUR WOODRUFF, and MATTHEW WOOLHOUSE[1] \\ University of Cambridge
}

\begin{abstract}
We review the paper by Andrew Clay McGraw, noting that it represents an interesting and valuable contribution to the study of music in cognition in its informed exploration of non-western musical perceptions. We raise a number of concerns about the methods used, and make suggestions as to how the issues that were empirically addressed in the paper might have been tackled in ways that would have enhanced the interpretability of its findings.
\end{abstract}

Submitted 2008 April 5; accepted 2008 April 14.

KEYWORDS: experimental method, non-western music, collaborative research

THIS fascinating paper takes as its starting point the idea that time in music and time in general, may be bound by culture in our conceptions, in particular, by processes of cross-domain mapping of the types that have been postulated as operational by ethnomusicologists, music theorists, and music psychologists. It explores by means of three experiments Becker's hypothesis that Balinese gamelan music reflects and perhaps embodies emic conceptions of time. The first uses an experimental paradigm known as the Implicit Association Test to investigate, by means of response times, the strengths of associations between musical and/or dance terms, music and/or instrument terms, and time and/or position terms, among Balinese musicians. It found evidence for a slight tendency for time-in music to be 'implicitly iconic' of time-in-general, though noted that the results showed high variability. The second experiment explored Balinese musicians' abilities to identify objective durations while listening to gamelan music; it found that subjects tended to rate examples as somewhat shorter than they actually were, a finding that contrasts with the results of a limited study of American musicians unfamiliar with gamelan who judged examples as rather longer than their actual durations. A final experiment investigates Balinese musicians' judgments concerning tempo transformations (rallentandi), finding that these listeners' preferences were equally for patterns of tempo change that previous literature suggested should be regarded as complex or 'unnatural.' These findings are discussed in the light of measurements of Balinese gamelan rallentandi that illustrate the complex, 'terraced' nature of closural tempo change that appears to predominate in this music.

We must say at the outset that this is precisely the type of research that requires to be done; it takes empirical methods that have been shown to have value in elucidating the dynamics of mind in western laboratory contexts and employs (and adapts) these to explore 'cognition in the wild' in a non-western cultural context. It poses questions about relationships between emic and etic conceptions of time and music, and about the real-time experience of temporal structures in music among Balinese musicians, from what appears to be a background of real understanding of, and engagement with, the musical culture that is being explored. The provisional nature of the results, and many of the limitations of the experimental design employed, are acknowledged. The response that the paper provokes is one of admiration, and any criticism that follow in this comment should be viewed as constructive and offered with the intent of precipitating debate about just how research of this type can, or should, be conducted.

To start with, while the author himself notes that he is 'not a psychologist', some assistance in designing the experiments and in analyzing and presenting the results would have been helpful. The 'readability' of the paper might have been aided by consulting on appropriate methods for presenting results. For example, Table 1 might have been presented as a boxplot, which would have aided a reader greatly, and it might be helpful to have the excerpts from experiment 2 available online for reference. The lack of statistical analysis of the results severely limits their interpretability; as they stand they are indicative and suggest lines for future research (of considerable value in itself), but the author's arguments 
would have been strengthened considerably by more rigorous consideration of experimental design and analysis of findings. For example, we find that the results from experiment three appear most convincing, but the absence of statistical analyses (it appears as though the data would be compatible with the application of the chi-square procedure) must lead a reader to suspend judgment somewhat.

Similarly, in experiment two, the 'noisiness' of the data might have arisen because each trial was unique, with all material being presented once only; had material been repeated, or had material been grouped into similar classes in some principled way, the data might have yielded clearer, more interpretable, results. Of course, following either route would have increased the number of trials, and probably tested the patience of the participants at least as much as their durational acuity! However, the use of the particular set of materials that were employed in experiments two and three (but particularly in experiment two) rather suggests that the author was seeking to explore 'the music itself' rather than the operationally defined, conceptually reduced, version of the music that would typically be required by the exigencies of a controlled experiment. The parameters in respect of which the musical materials used were varied - the dependent variables - needed to be spelled out more explicitly and separated out, insofar as was possible, from other aspects of the music that were not subject to experimental control, in order for the experimental results to afford the type of information usually required of an experiment on musical cognitions.

A further criticism that could be raised is that context of the experiments needed to be more highly controlled; it is evident from Figure 3 that experiment two, the duration perception test, was a group experience and it seems quite feasible that a degree of participant interaction may have occurred that could have impinged on the results. However, the only one of us who has practical experience in conducting experiments on music cognition in non-western contexts (LS-in South Korea and in northern Mozambique) is highly aware of the difficulties in such contexts of attempting to control for extraneous factors that might impact on task performance. Moreover, it is quite possible that requiring participants to undertake the tasks isolated from others might have diminished the ecological validity of the experiments. Perhaps more compelling reasons for group testing are to be found in the practical problems that may arise if an experimenter in a non-western context, typically on a tight time budget, attempts to test individuals separately; participants may perceive the experimenter as requiring them to make unreasonable time commitments determined solely by the experimenter's own schedule.

This brings up what appears to us to be an interesting point that we raise as a possible topic for discussion. The typical western laboratory procedure, where an experimental participant is tested in isolation (often in a sound-proof booth), is usually taken as likely to produce results that are reasonably generalizable to the broader cultural contexts in respect of which the capacity being tested is employed in everyday life. It might be the case that in domains (such as music) where the paradigmatic experiential mode in a given culture may be interactive rather than individualistic, the bases for some types of judgment are as much consensual as they are individualistically perceptual. In other words, it may be that some types of musical judgment are necessarily exercised as social and interactive behaviors rather than being evidenced in acts of individual judgment, requiring to be explored both individually and by means of analyses of group processes and outcomes which could be undertaken with the aid of video.

Returning to the substance of the paper, we were concerned by the use of the IAT test (as, indeed, was the author, who acknowledges that the test "is based upon the rapid cognition of words, not of music itself"). In many respects this test seems to presuppose a model of semantic, or conceptual-intentional, memory that is not a million miles from that proposed by Collins and Quillian in 1969, where individual concepts or features are represented as more, or less, proximate nodes in a network. While this may be a viable model for at least some aspects of lexical memory, it seems likely to have limited applicability to domains such as music where at least some information is fundamentally non-verbal and perhaps nonverbalizable. It is not clear precisely what type of task might be most appropriate to uncover the potential connections (if any) between the experience of music, the materials of the discourse that has developed around it and the general linguistic and conceptual framework in which that discourse is embedded. But a focus solely on the linguistic domain alone appears to us unlikely to be capable of revealing much that is consistent or stable concerning the dynamics of musical culture.

We also felt that there were aspects of a number of literatures that might have been either referenced or addressed to the benefit of the paper's arguments. For example, reference to the work of Zbikowski (2002) might have broadened the scope of the discussion of cross-domain mapping and music. Similarly, reference to the work of Narmour $(1989,1992)$ might have enriched the discussion of the experience of musical time by considering ways in which the 'microflux' of the experience of music has 
been theorized as being modulated by the structure of the music. In general, previous empirical research that has sought to explore cross-cultural cognitions is not too well-represented here. Useful summary accounts can be found in various entries in the MIT Encyclopedia of Cognitive Science (1999), and might have helped the author frame more explicitly some of the conceptual issues that he addresses, and volumes such as Bradd Shore's (1996) Culture in Mind would certainly have provided much more substantial foundations for the conceptual frameworks that underpin this paper. An instance is found in the author's caveat in footnote xix, that "such a thing as the unconscious exists and that it functions in the same way cross-culturally." This suggestion begs a number of questions and its bases might have been clarified had a wider range of previous literature been explored.

The paper might also have benefited from the provision of more ethnographic content. It would have been useful to know, for example, how the participants in the experiments felt about being required to perform the experimental tasks. The Balinese composer shown in Figure 2 certainly appears wholly at ease with the task, but the experiences of older participants, or perhaps of participants from more rural contexts (if any were included) might have as much shaped by the context of the task as by the task itself. Similarly, a more dense account of the social contexts within which the experiments were undertaken might have been informative for a reader in evaluating the ways in which the experimental tasks were construed by participants as social actions rather than individual obligations. Indeed, a more vivid impression of the author's central theme could have been provided by examining more closely those features of Balinese music thought to be associated with either cyclic or non-linear concepts of time. The author makes an interesting reference to the wave metaphor-ombak-in the Balinese conception of music. However, other distinctive aspects such as the colotomic organization of the gamelan, the use of interlocking patterns and cyclic rhythms, and considerations such as the length of performance (Wayang shows can extend throughout the night) might have provided valuable detail serving to contextualize the empirical and theoretical aspects of the discussion.

Ultimately, the extent to which this paper provides insights into the minds of Balinese musicians must be tested against the criteria proposed by Cole (1996, p. 226), following Bronfenbrenner (1979). Cole (1996) suggests that there are three identifiable criteria for 'ecologically valid research': it must (i) maintain the integrity of the real-life situations it is designed to investigate; (ii) be faithful to the larger social and cultural contexts from which the subjects came; and (iii) be consistent with the participants' definition of the situation, by which Cole means that the experimental manipulations and outcomes must be "perceived by the participants in a manner consistent with the conceptual definitions explicit and implicit in the research design.” This paper appears to go a considerable way towards meeting those criteria; if it fails to meet them entirely, that failure is shared by most other papers in the literature. However, the weaknesses and strengths of the paper do point up a further need, that for collaboration between ethnomusicologists and those involved in studying music cognition. At present, our knowledge of the musical mind is largely based on results derived from laboratory-based studies of western trained or encultured listeners and performers. We urgently need to know how that knowledge may be constrained by —or even whether its informativeness may be limited to - the social and cultural processes and systems from which it emerges. Only by collaborating across disciplines-a course of action that is likely to be messy and contentious but which the example of this paper suggests is both possible and necessary - can we begin to understand just what it is that we actually know about music in mind.

\section{NOTES}

[1] Science \& Music Group, Centre for Music \& Science, Faculty of Music, University of Cambridge.

\section{REFERENCES}

Bronfenbrenner, U. (1979). The ecology of human development: Experiments by nature and design. Cambridge, MA: Harvard University Press

Cole, M. (1996). Cultural psychology. London: Belknap Press of Harvard University Press. 
Collins, A. M., and Quillian, M. R. (1969). Retrieval time from semantic memory. Journal of Verbal Learning and Verbal Behavior, 8, 240-247.

Wilson, R. A. \& Keil, F. C. (Eds.) (1999). MIT encyclopedia of cognitive sciences. Cambridge, MA: MIT Press.

Narmour, E. (1989). The analysis and cognition of basic melodic structures. London: University of Chicago Press.

Narmour, E. (1992). The analysis and cognition of melodic complexity. London: University of Chicago Press.

Shore, B. (1996). Culture in mind: Cognition, culture, and the problem of meaning. Oxford: Oxford University Press.

Zbikowski, L. M. (2002). Conceptualizing music: Cognitive structure, theory and analysis. Oxford: Oxford University Press. 